\title{
Web Camera Use in the Neonatal Intensive Care Unit: Impact on Nursing Workflow
}

\author{
Aditya Joshi, MD; Po-Huang Chyou, PhD; Zoya Tirmizi, MD; \\ and Jody Gross, MD
}

\begin{abstract}
Background: Many neonatal intensive care units (NICU) are using web camera systems to allow virtual visitation of the infant by family members. Generally, families appreciate the web camera and utilize this service. However, no one has looked into the change on nursing workload after implementation of a web camera.
\end{abstract}

Objective: This study was designed to explore the perception of nurses and their workflow and identify determinants that may disrupt or facilitate the use of a commercially available camera service. Our primary goal was to see if the camera system interferes with the nursing care.

Study Design: This was a prospective, questionnaire-based study conducted between March and September 2014. Parents were offered the camera service and signed a consent form before use. Parents who refused the camera were the group designated as "off camera." There were two infant groups; one cared for using the cameras and the other not. The camera service was used continuously during the study period, except during procedures, baby care, and feedings. Demographic information on nursing staff and neonates was collected weekly. Questionnaires were placed at each bedside for nurses to complete on each shift for each infant once weekly. The questionnaires for infants off-camera did not have questions regarding the camera. However, the rest of the questions were similar regarding time spent interacting with family members, face to face, or on the phone. Data on time spent manipulating the camera per shift were also obtained.

Results: Surveys for 623 on-camera and 130 off-camera infants were completed by 42 nursing staff. Findings showed that caring for multiple infants while using the web camera increased nurses' workload and stress, which they perceived as having an adverse effect on the ability to provide quality care. Family decisions not to utilize the camera service were mainly cultural, and noted among Mennonite, Amish, and Hmong families. Some families residing close to the hospital also preferred visiting the baby personally rather than using the camera service.

Conclusion: Parents and nurses perceived web cameras as beneficial in the NICU setting. However, nurses spent significant time manipulating cameras and addressing parental concerns over the phone, causing disruption in their workflow, which had the potential to decrease quality of care for infants. To compensate for these disruptions, we recommend increasing awareness of the potential issues with both cameras and families and providing multiple training sessions to nursing staff before systems are implemented.

Keywords: Neonatal intensive care unit; Neonatal nursing; Web camera

Corresponding Author: Jody Gross, MD, Marshfield Clinic/St. Joseph's Hospital, Department of Neonatology, 1000 N Oak Avenue, Marshfield, WI 54449, USA. Email: gross.jody@marshfieldclinic.org

\author{
Received: March 20, 2015 \\ Ist Revision: July 15, 2015 \\ 2nd Revision: October 14, 2015 \\ Accepted: October 28, 2015
}

doi: $10.3121 / \mathrm{cmr} .2015 .1286$ 
ore and more neonatal intensive care units (NICU) systems to facilitate family bonding with sick infants. ${ }^{1,2}$ The goal of camera services is to allow virtual visitation of the infant by family members and to enhance bonding. Parents have shown a positive attitude towards the camera service. ${ }^{1-3}$ These technologies need to be user friendly in order to minimally impact the workload of nursing staff. ${ }^{2}$ Poorly planned implementation could lead to underutilization of the camera service. $^{2}$

The web-based camera service allows parents and other relatives to visualize the baby admitted in the NICU through a password protected system. The web camera is attached to the bedside of the baby; any baby admitted to the NICU can be accessed by the cameras following parental written consent. Each family is issued an individual password to login to the secure system. Families are able to share the password with their friends and relatives at their discretion. The nurses taking care of the baby are also responsible for checking that the camera's focus is centered on the infant, and that it is switched off during procedures, physical assessment, and bathing, and that it is then switched back on afterward. There is a telephone at every infant bedside, so when parents call, the nursing staff are available to answer questions and concerns.

Hawkes et $\mathrm{al}^{2}$ evaluated nursing staff perception prior to implementation of a web camera service in which a majority of nurses revealed a negative attitude; $72 \%$ of the nurses feared increased workload after the camera service started. Nurses were also concerned about security issues, an increased number of phone calls, maintaining the camera system, and the infant being seen in the view. ${ }^{2}$ Angel Eye developers, a camera company, interviewed nurses after the camera service was implemented. There were concerns regarding the design of the camera and ease of use. ${ }^{1}$ Rhoads et al ${ }^{1}$ were concerned that if the cameras were implemented haphazardly then there would be increasing resistance from the NICU staff. Piecuch et $\mathrm{al}^{4}$ noted that the increased frequency of phone calls by the video mothers compared to the control mothers might have caused distress to the nurses. There are limited data on change in workload after implementation of a camera service.

It is important to understand the workload and stress factors of NICU nurses in order to prevent adverse events. Multiple factors like patient acuity, staffing and management factors, highly technical environment, death, family needs, and ongoing need for crisis intervention and decision making are implicated as stressors. ${ }^{5}$ Tucker et $\mathrm{al}^{6}$ noted that infant mortality in the NICU increased with an increase in nursing staff workload. Increased workload can, thus, adversely affect patient safety. ${ }^{7}$ Therefore, assessment of the impact of any new technology on nursing work load is essential. Filho et al ${ }^{8}$ examined the possible association between the intensity of staff workload and adverse events in the NICU like accidental extubation, obstruction of endotracheal tube, and accidental disconnection of ventilator circuit. Adverse events were noted frequently during their 6-month study period. The more infants cared for per nurse, the more likely was the occurrence of intermediate adverse events. ${ }^{8}$

We evaluated the implementation of a web camera service in our NICU. Our study hypothesis was that implementation of the web camera service would increase nursing workload. We studied this by having nurses document their time spent dealing directly with the camera system for each infant on camera during their shift. We also questioned nurses about time spent with families as a direct result of the camera. We attempted to identify determinants that might disrupt or facilitate the use of camera service among nursing staff. We

Table 1: Demographic characters of the nurses and infants participated in the NICVIEW camera study.

\begin{tabular}{lcc}
\hline & \multicolumn{2}{c}{ Nurses working } \\
\cline { 2 - 3 } Characteristics & On Camera & Off Camera \\
\cline { 2 - 3 } Median number of years worked in NICU & 15 & 15 \\
Median infants completed weeks of gestation at birth & 32 & 34 \\
Median Infants completed gestation at survey date & 35 & 35 \\
Average number of work hours per week (\%) & & 16.9 \\
$<32$ hours & 16.2 & 82.3 \\
$32-40$ hours & 82.3 & 0.8 \\
$>40$ hours & 1.4 & \\
Number of infants cared for per shift (\%) & & 9.2 \\
One & 2.6 & 72.3 \\
Two & 64.5 & 17.7 \\
Three & 30.5 & 0.8 \\
Four & 2.4 & \\
\hline
\end{tabular}

2

Web Camera use in the NCIU

$C M \& R 2016$ : 1 (March) 
Table 2: Spearman's correlation coefficient between numbers of years worked in NICU with other variables.

\begin{tabular}{lcc}
\hline & Correlation coefficient (r) & $\boldsymbol{P}$ value \\
Number of infants cared for per shift on camera & -0.04 & 0.28 \\
Number of infants cared for per shift off camera & -0.07 & 0.44 \\
Number of infants per shift for all nurses & -0.05 & 0.194 \\
Time spent per shift manipulating camera & 0.22 & $<0.001$
\end{tabular}

further examined the parental perception and use of the camera service based on their interaction with the nursing staff.

\section{Methods}

Study Site and Sample

A prospective, questionnaire-based study was conducted at the Marshfield Clinic/St Joseph Hospital, Wisconsin, a tertiary pediatrics hospital with 24 NICU beds, 42 nursing staff, and an annual admission of 350 infants. The NICU camera service was first implemented in December 2013. The study was approved by the Marshfield Clinic Institutional Review Board.

The questionnaire survey activities took place between March and September, 2014. The weekly survey targeted a 24-hour period covering two to three nursing shifts, inclusive of 42 nursing staff. Two survey questionnaires were designed; one for the nurses taking care of infants on camera and another for nurses caring for infants off camera. The face and content validity of the draft questionnaire was assessed individually by experts in the field of neonatology as well as by researchers, statisticians, and nursing supervisors [The questionnaire can be obtained on request from the corresponding author]. Nurses completed the questionnaire based on status of camera use as well as on their interaction with parents. Parents did not complete survey forms; however, parental perception about the camera service was obtained based on their comments to nursing staff. Nurses completed a survey for each infant they cared for each shift, so if they cared for two infants on camera and one not on camera, they would complete three surveys.
The survey tool collected demographic data on the infant and on nursing staff, including the infant's gestational age the day of the survey, the length of the nurse's shift, the number of years the nurse worked in the NICU setting (experience), and the number of infants cared for that day. The survey included their perception on the usefulness of the camera service on a Likert scale, time spent manipulating the camera and interacting with the family for the concerns based on camera images, and an open-ended question focused on their perception with respect to camera at the end. Respondents assigned ratings based on a 5 point Likert scale, 1 (strongly disagree) to 5 (strongly agree). Occasional parental comments were noted by the nursing staff and reported on the survey sheet. The number of logins by the parents or other family members every month was captured. Severity of the illness of an infant was measured on the basis of their gestational age. Survey forms for infants off camera included demographic data on the baby and nursing staff and time spent interacting with family members. Time spent interacting with family members for infants on and off camera was compared.

Comparisons between response from nurses caring for infants on and off camera were performed using the Chi-square test for categorical variables and the Wilcoxon rank sum test for continuous variables. In addition, the Spearman's correlation coefficients were obtained for assessing the relationships with nurses' perceptions on usefulness of the camera and time spent manipulating the camera. $P$ values were derived from Chi-square and Wilcoxon rank sum analysis. A $P$ value of $<0.05$ was considered statistically significant. All data

Table 3: Spearman's correlation coefficient of nurse's perception on usefulness of camera service with other variables.

\begin{tabular}{lccc}
\hline Number of infants on camera per shift & $\begin{array}{c}\text { Number of } \\
\text { of surveys (N) }\end{array}$ & \multicolumn{2}{c}{$\begin{array}{c}\text { Correlation } \\
\text { coefficient (r) }\end{array}$} \\
\hline One & 15 & 0.36 & 0.17 \\
Two & 402 & 0.2 & 0.17 \\
Three & 190 & 0.65 & 0.02 \\
Four & 15 & 0.20 & 0.009 \\
Hours worked per shift & 623 & 0.05 & 0.19 \\
Infants completed weeks of gestation at birth & 623 & 0.01 \\
\hline
\end{tabular}


analyses were carried out using a commercially available statistical software package (SAS version 9.3, English, Cary, NC, USA).

\section{Results}

All 42 nurses participated in the study. Survey response rate was $82 \%$ of the total possible 918 surveys. Data analyzed included 753 surveys (623 on-camera and 130 off-camera.) As shown in table 1, demographic characteristics of the nurses and infants captured on and off camera were comparable. The percentage of nurses caring for three or four infants on camera was higher compared to nurses caring for three or four infants not using the camera. There was a significant positive correlation between number of hours worked and nurse's perception that the camera service was beneficial for the families. This relationship was noted to be strongest when the number of infants being cared for was highest $(n=4$, table 3$)$.

A significant amount of time was spent manipulating camera and interacting with the family members for the concerns seen on the camera (table 4). There was a statistically significant correlation between the number of infants nursed and increase in time spent on camera manipulation (table 2, $\mathrm{r}=0.22, P<0.001)$. The percentage of nurses spending $15-30$ minutes manipulating the camera were $29 \%, 33 \%, 26 \%$, and $45 \%$ respectively for one, two, three, and four infants being cared for. On the other hand, the percentages of nurses spending 31-60 minutes manipulating the camera were much lower $(<10 \%)$. With these two categories combined, the percentages of nurses spending 15-60 minutes manipulating the camera were $31 \%, 39 \%, 29 \%$, and $54 \%$ respectively for one, two, three, and four infants being cared for. Due to the small number with nurses caring for four infants, these differences were not statistically significant $(P=0.1204)$. However, it appears that the more infants cared for on camera, the more time it took.

Severity of illness of an infant was not significantly associated with time spent manipulating the camera. Among surveyed nurses, $61.2 \%$ reported that they always switch off the camera during procedures and physical assessment. A statistically significant association was found between nurses working more than 40 hours per week and forgetting to switch off the camera during procedures.

Reduction in parental anxiety with camera use was reported by $64.6 \%$ of nurses, while $3.4 \%$ reported increased parental anxiety. Regular camera use by parents was noted in $64.7 \%$ of surveys, and $5.9 \%$ of families called the NICU for concerns seen during camera use. Whereas $15.4 \%$ of nurses reported spending greater than 60 minutes interacting with the family over the phone, only $8.5 \%$ of surveyed nurses working with off-camera infants reported greater than 60 minutes of interaction with family members. A statistically significant, but weak positive correlation, was noticed $(r=0.1)$ between severity of illness of the baby on camera and time spent interacting with the family.

Based on the interaction with the parents and their feedback, $72.9 \%$ of nurses believed that having the camera service for parents is useful. Determinants for refusing the camera service were primarily cultural, economic, and distance of residence from the hospital. Amish, Mennonite, and Hmong families preferred not to use the camera services. Some families living close to hospital also preferred visiting baby at hospital rather than using camera services. However, most of the families who were staying close by at the Ronald McDonald House wanted the camera service, both for themselves and for distant family members.

Feedback from nurses on open-ended questions highlighted several issues. Moving the camera to a different place, like from the incubator to an open crib, was time consuming. Whenever the camera stand moved, it disturbed the focus on the baby. The camera was also perceived to be unstable when mounted to the side of an open crib. Some nurses also felt that they were being checked up on by parents and felt like a "babysitter." Nurses also wished the on/off button was on the side of the camera where they could easily see it, rather than where it was positioned facing the baby. Mothers selfreported increased breast milk volumes while observing their baby on camera. Though no major adverse events were noted during our study period, one camera did fall, creating the

Table 4: Time spent manipulating camera service and interacting with the family members for babies on camera.

\begin{tabular}{lcr}
\hline Time spent ( minutes) & $\begin{array}{c}\text { Manipulating cameras / } \\
\text { number of surveys }\end{array}$ & $\begin{array}{c}\text { Interacting with family members / } \\
\text { number of surveys } \\
\text { None }\end{array}$ \\
$<15$ & $17(2.7 \%)$ & $117(18.8 \%)$ \\
$15-30$ & $371(59.6 \%)$ & $84(13.5 \%)$ \\
$31-60$ & $195(31.3 \%)$ & $114(18.3 \%)$ \\
$>60$ & $30(4.8 \%)$ & $96(15.4 \%)$ \\
Total & $10(1.6 \%)$ & 623 \\
\hline
\end{tabular}

$4 \quad$ Web Camera use in the NCIU

$C M \& R 2016$ : 1 (March) 
potential to injure the patient. That event caused the NICU to permanently mount the camera units to each headwall, rather than attach them to each warmer, crib, or bassinet. Nurses were also concerned that time spent manipulating camera services might take away time from other sick neonates, which could ultimately lead to an adverse event.

\section{Discussion}

Our hypothesis that implementation of the camera system increased the real workload among the nursing staff was supported by the data. The nurses spent a greater amount of time manipulating the camera and interacting with the family members for concerns seen on the camera compared to nurses attending off-camera infants. In a critically important setting like the NICU, increased nursing work load can have a negative impact on quality of care.

Our results on parental perception were consistent with previous studies. ${ }^{1,9}$ Parents were noted to be using the service on a regular basis. We further noted that family members residing outside the United States also frequently used the camera service, proving helpful for connecting with distant family members and giving new meaning to the term 'familycentered care.'

Implementing a new technology often progresses through different phases, including dissemination, adoption, implementation, and continuation. ${ }^{10,11}$ Determinant analysis at all of these stages is important, because if not conducted, full implementation of the new technology may fail. Understanding the characteristics of the users (in our case nurses) and the innovation (the web camera) is important along with understanding the sociopolitical context and characteristics of the organization.

There are reports of web cameras initiated without thorough prior investigation regarding the NICU nurse and family needs. The outcome was underutilization of the service, because the cameras were difficult to maneuver, and the website was cumbersome. ${ }^{3}$ An important goal would be to create a positive attitude from the nursing staff towards the web camera service. To provide optimal care for infants, it is important to have a thorough understanding of the potential barriers, in order to consider them when designing interventions. In our study, nurses with more work experience were noted to spend more time manipulating the camera. This could possibly be due to discomfort with a new technology. More experienced nurses are more likely to have a wellestablished work-flow pattern and may be less accepting of a new technology. In these instances, new nursing staff could act as role models for those who have less experience with new technologies, and then later they can be integrated into the system. Increased time spent managing malfunctioning cameras was reported several times and was a source of frustration to the nurses. Therefore, it is important to train nurses on the technology before implementation of the camera. Nursing administration also needs to be more proactive, seeking alternative measures for handling increased phone calls from parents, which distract nurses from accomplishing other patient-care duties.

There were several concerns with the design of the camera. Transitioning the camera to the open crib from the incubator and adjusting the camera to the crib was considered time consuming by nurses. Some nurses commented that they felt like a babysitter when caring for on-camera infants. Educating nurses more on the perceived usefulness of the camera can be helpful. It is also important to involve the nurses before implementation of these kinds of services, as was done at our institution.

Our study has several limitations. The small sample size of the study group with only 42 nursing staff limits the study power. Follow-up time was limited in duration after camera installation, and data were obtained at only a single institution. Because nurses completed the survey multiple times during the study period, only initial completion of surveys were independent, with potential for introduction of bias associated with repeated survey completion. Another limitation of this study is that we looked only at nursing staff and did not include physicians. Hawkes et $\mathrm{al}^{2}$ looked into physician perception before implementation of a camera service and noted that $20 \%$ of physicians felt that web camera service would increase the staff workload.

Our study supported the concept that the web based camera service was very useful for parents when it was not possible for them to visit the NICU. In a tertiary rural hospital where parents are geographically distant, the web camera service is a good alternative to promote bonding. Further prospective studies are needed to examine the impact of web camera service on the duration of the hospital stay and of transitioning care to home.

Over time, as nurses had more exposure to caring for an increasing number of infants on camera, the perception of the service was viewed as beneficial. Findings in this study also showed that simultaneously caring for multiple infants while using the web camera increased nurses' workload and stress, which they perceived as having an adverse effect on quality of infant care. Therefore, to allow the nurses to compensate for these disruptions, we would recommend increasing awareness of the potential issues with both cameras and families, multiple training sessions to the nursing staff before the systems are implemented. 


\section{Acknowledgments}

The authors thank the Marshfield Clinic Research Foundation's Office of Scientific Writing and Publication for technical assistance in the preparation of this manuscript.

\section{References}

1. Rhoads SJ, Green AL, Lewis SD, Rakes L. Challenges of implementation of a web-camera system in the neonatal intensive care unit. Neonatal Netw. 2012;31(4):223-228.

2. Hawkes G, Livingstone V, Ryan C, Dempsey E. Perceptions of webcams in the neonatal intensive care unit: here's looking at you kid! Am J Perinatol. 2015;30(02):131-136.

3. Yeo C, Ho SK, Khong K, Lau Y. Virtual visitation in the neonatal intensive care: experience with the use of internet and telemedicine in a tertiary neonatal unit. Perm J. 2011;15(3):32-36.

4. Piecuch R, Roth RS, Clyman R, Sniderman SH, Riedel PA, Ballard RA. Videophone use improves maternal interest in transported infants. Crit Care Med. 1983;11(8):655-656.

5. Heuer L, Bengiamin M, Downey VW, Imler NJ. Neonatal intensive care nurse stressors: an American study. Br J Nurs. 1996;5(18):1126-1130.

6. Tucker J; UK Neona tal Staffing Study Group. Patient volume, staffing, and workload in relation to risk-adjusted outcomes in a random stratified sample of UK neonatal intensive care units: a prospective evaluation. Lancet. 2002;359(9301):99-107.

7. Carayon P, Gurses AP. Nursing workload and patient safety-A Human factor engineering perspective. In: Patient Safety and Quality: An Evidence Handbook for Nurses. Hughes RG, ed. Rockville, MD: Agency for Healthcare Research and Quality; 2008.

8. Lamy Filho F, Silva AA, Lopes JM, Lamy ZC, Simões VM, Dos Santos AM. Staff workload and adverse events during mechanical ventilation in neonatal intensive care units. J Pediatr (Rio J). 2011;87(6):487-492.

9. Heermann JA, Wilson ME, Wilhelm PA. Mothers in the NICU: outsider to partner. Pediatr Nurs. 2005;31(3):176-181, 200.

10. de Veer AJE, Fleuren MAH, Bekkema N, Francke AL. Successful implementation of new technologies in nursing care: a questionnaire survey of nurse-users. BMC Med Inform Decis Mak. 2011;11(1):67.

11. Fleuren M, Wiefferink K, Paulussen T. Determinants of innovation within health care organizations: Literature review and Delphi study. Int J Qual Health Care.

2004;16(2):107-123.

\section{Author Affiliations}

Aditya Joshi, MD*; Po-Huang Chyou, $\mathrm{PhD}^{*}$;

Zoya Tirmizi, MD*; Jody Gross, MD

"Department of Pediatrics, Marshfield Clinic/St. Joseph's Hospital, Marshfield, Wisconsin

'Biomedical Informatics Research Center, Marshfield Clinic Research Foundation, Marshfield, Wisconsin

${ }^{*}$ Department of Neonatology, Marshfield Clinic/ St. Joseph's

Hospital, Marshfield, Wisconsin 